\title{
Zur Kenntniss der Reaction zwischen Chloralhydrat und Alkali;
}

von

\author{
W. Böttger und A. Kötz.
}

Einleitung.

Bei Beginn dieser Untersuchung bestand ursprünglich die Absicht, die Gesetzmässigkeiten für einige analoge Reactionen, wie die Bildung von Benzil- resp. Isatinsäure aus Benzil resp. Isatin und Alkalihydroxyd zu ermitteln. Die Frage, die wir zunächst zu beantworten wünschten, war die nach der Beziehung zwischen der Concentration des Hydroxylions und der Geschwindigkeit des Vorgangs. Die Vorversuche, die wir zunächst mit Benzil, und zwar wegen der geringen Löslichkeit desselben in alkoholischer Lösung vornahmen, ergaben jedoch einen nicht klar übersehbaren Verlauf, sodass wir uns dem Studium des einfacheren Falles, der Wechselwirkung zwischen Chloralhydrat und Alkali unter Bildung von Chloroform und Formiat zugewendet haben.

Für diese Reaction bestehen nach den Untersuchungen von Reicher ${ }^{1}$ ) unaufgeklärte Unbestimmtheiten ${ }^{2}$ ) über die Ordnung der Reaction, welche vermuthen lassen, dass es sich hierbei um Vorgänge secundärer Natur handelt. Der Aufklärung dieser Verbältnisse haben wir uns unterzogen, und im Nachstehenden soll über die oder wenigstens einen der maassgebenden Gründe für die Abweichungen von dem einfachen Reactionsschema berichtet werden.

1) L. Th. Reicher, Rec. Pays-Bas, 4, 347, 1885; nach Maandbl. v. Naturw, 12, 78 (1885).

2) W. Ostwald, Lehrb. der allg. Chemie, 2. Bd., II, 242. 
482 Böttger u.Kotz: Reaction zw. Chloralhydrat u. Alkali.

\section{Versuche.}

Die hier mitgetheilten Versuche wurden bei $25^{\circ}$ ausgeführt; von der Angabe anderer Versuche, die bei $6^{0}$ gemacht wurden, und die mehr einen orientirenden Charakter hatten, soll hier abgesehen werden, weil sie kein wesentlich anderes Bild zeigten wie jene. - Ueber die Ausführung der Versuche selbst ist zu bemerken, dass eine Lösung von Chloralhydrat von bekannter Concentration mit der Lösung einer bekannten Menge von $\mathrm{NaOH}$, welche die gleiche Temperatur wie erstere hatte, vermischt wurde. (Die im Gefässe bleibende Menge der Natronlauge wurde durch Titration bestimmt und die entsprechende Correktur angebracht.) Da somit das Gesammtrolum, das Volum and die Concentration der alkalischen Lösung bekannt waren, konnte der Anfangstiter berechnet werden, der wegen der raschen Umsetzung direct nicht bestimmt werden konnte.

Die Berechnung ist natürlich unter der Voraussetzung durchgeführt worden, dass sich mit der Vermischung nicht erhebliche Volumänderungen abspielen, welche die Genauigkeit des Anfangstiter wesentlich beeinflussen könnten. Uebrigens wird sich zeigen, dass bei der starken Abweichung der Constanten an ein Zurückführen auf derartige Unsicherheiten nicht zu denken ist. Durch einen Versuch, bei welchem das Alkali nicht zur Lösung des Chloralhydrats, sondern zu einem abgemessenen Volum Wasser gegeben wurde, überzeugten wir uns, dass der berechnete Titer mit dem thatsächlich gefundenen gut übereinstimmte.

Ueber die Zeitbestimmung ist $z \mathfrak{u}$ erwähnen, dass die Vermischung in 10 bis 15 Secunden ausgeführt wurde. Als Anfangspunkt wurde der Zeitpunkt gewählt, welcher um die Hälfte dieses Intervalles hinter dem des Beginnes der Mischung lag. Die Unsicherheit der Feststellung der Anfangszeit beträgt wohl im höchsten Falle 5 Secunden. Die Zeiten, zu welcher bestimmte Proben entnommen wurden, konnten bequem durch Verwendung einer Pipette von bekannter A usflusszeit ermittelt werden. Als der zu einer bestimmten Umsetzung gehörige Zeitpunkt wurde der um die halbe Ausflusszeit vermehrte Anfangswerth des Aus- 
flusses gewählt. Die Unsicherbeit der Anfangszeit tritt übrigens bei Zeiträumen über 5 Minuten zurück.

Die entnommenen Proben wurden in gemesseneVolume Salzsäure von bekannter Stärke entleert und darauf der Ueberschuss der Säure mit kohlensäurefreier Natronlauge unter Benutzung von Phenolphtalein als Indicator bestimmt. Zur Herstellung der Chloralhydratlösungen wurde Leitfähigkeitswasser benutzt und ausserdem wurde zur Einschränkung der Absorption der Kohlensäure auf raschen Verschluss der Gefässe Gewicht gelegt. Für die nicht umgesetzte Menge Chloralhydrat müsste eine Correctur in dem Sinne angebracht werden, dass die bei der Titration mit Alkali gefundene Säure um einen gewissen Betrag zu vergrössern ist, da Chloralhydrat wie eine ganz schwache Säure wirkt und demgemäss eine bestimmte, wenn auch kleine Menge Alkali neutralisirt. Die hierfür anzubringende Correctur ergab sich aus Titrationen von Chloralhydratlösungen von bestimmtem Gehalt zu $0,90 \mathrm{Cm}^{3}$ einer ${ }^{1} / 27,95 \mathrm{NaOH}$ für $20 \mathrm{Cm}^{3}$ einer $0,17 \mathrm{n}$-Lösung von Chloralhydrat. Die Titrationen sind, solange nennenswerthe Ueberschüsse von Chloralhydrat vorhanden sind, ausserdem mit einer durch die schwach saure Natur dieses Stoffes bedingten Unsicherheit behaftet. Aus dem Angeführten ergiebt sich, dass die Versuchsfehler besonders in den ersten Stadien einer Reihe ziemlich gross sein müssen.

Es sollen nun zunächst zwei Parallelversuche angeführt werden, die dazu benutzt wurden, die Anwendbarkeit eines der Reactionsgesetze auf diesen Vorgang zu prüfen. Die Bedeutung der Zahlen in den einzelnen Columnen ist ohne weiteres zu erkennen. In Tabelle 1 a steht unter $A-x$ resp. $B-x$ die zu verschiedenen Zeiten in $22,72 \mathrm{Cm}^{3}$ des Reactionsgemisches vorhandene Menge $\mathrm{NaOH}$ resp. Chloralhydrat, ausgedrückt in Cm. ${ }^{3}{ }^{1} / 22,08$ äquivalent-normaler Lösungen. In Tabelle $1 \mathrm{~b}$ sind die Mengen angegeben, die in $20,02 \mathrm{Cm}^{3}$ enthalten sind.

\section{Tabelle 1a.}

Angewendete Menge Chloralhydrat 0,8422 Grm. in $299,3 \mathrm{Cm} .{ }^{3}$

Angewendete Menge $\mathrm{OH} 0,08923 \mathrm{Grm}$. in $299,3 \mathrm{Cm}^{3}$

Verhältniss in Verbindungsgewichten $\frac{\mathrm{NaOH}}{\mathrm{CCl}_{3} \cdot \mathrm{CO}_{2} \mathrm{H}_{3}}: \frac{1}{0,970}$. 
484 Bottger u.Kotz: Reaction zw. Chloralhydrat u. Alkali.

$\begin{array}{rccccc} & \text { Min. } & A-x & B-x & \boldsymbol{K}_{1} & K_{2} \\ \text { 1. } & 0 & \mathbf{7 , 7 5} & \mathbf{7 , 5 2} & - & - \\ \text { 2. } & 1 & \mathbf{6 , 2 1} & \mathbf{5 , 9 8} & 0,229 & 14,6 \\ 3 . & 2,5 & \mathbf{4 , 7 3} & \mathbf{4 , 5 0} & \mathbf{0 , 2 0 5} & 15,0 \\ \text { 4. } & \mathbf{4 , 7} & \mathbf{3 , 5 0} & \mathbf{3 , 2 7} & 0,1 \mathbf{7 5} & 16,2 \\ \text { 5. } & 11,7 & \mathbf{1 , 5 4} & 1,31 & \mathbf{0 , 1 4 9} & \mathbf{2 1 , 5} \\ 6 . & 19,7 & 1,18 & \mathbf{0 , 9 5} & \mathbf{0 , 1 0 5} & \mathbf{1 8 , 2} \\ \text { 7. } & 31 & 0,77 & \mathbf{0 , 5 4} & \mathbf{0 , 0 8 5} & 20,1 \\ 8 . & 59 & \mathbf{0 , 4 6} & \mathbf{0 , 2 3} & - & \mathbf{2 1 , 5} \\ 9 . & 210 & \mathbf{0 , 1 3} & - & - & - \\ 10 . & \infty & 0,04 & - & - & -.\end{array}$

Tabelle $1 \mathrm{~b}$.

Angewandte Menge Chloralhydrat 0,8422 Grm. in $299,5 \mathrm{Cm}^{3}$ Angewandte Menge OH $0,08923 \mathrm{Grm}$, in $299,5 \mathrm{Cm}^{3}$

Verbältniss in Verbindungsgewichten $\frac{\mathrm{NaOH}}{\mathrm{COl}_{3} \cdot \mathrm{CO}_{2} \mathrm{H}_{3}}=\frac{1}{0,970}$

$\begin{array}{rccccc} & \text { Min. } & A-x & B-x & K_{1} & K_{2} \\ \text { 1. } & 0 & 8,80 & 8,54 & - & - \\ \text { 2. } & 1 & 7,26 & 7,00 & 0,199 & 12,5 \\ \text { 3. } & 4,7 & 4,00 & 3,74 & 0,174 & 15,1 \\ \text { 4. } & 11 & 2,21 & 1,95 & 0,134 & 16,7 \\ \text { 5. } & 17,3 & 1,47 & 1,21 & 0,113 & 18,4 \\ 6 . & 29,7 & 0,90 & 0,64 & 0,087 & 20,1 \\ \text { 7. } & 59 & 0,51 & 0,25 & - & 29,2 \\ 8 . & 102 & 0,28 & 0,02 & - & - \\ 9 . & 262 & 0,13 & - & - & - \\ 10 . & \infty & 0,05 & - & - & -\end{array}$

Zur Berechnung der unter $\boldsymbol{K}_{1}$ angegebenen Werthe dienten die Zahlen unter $\boldsymbol{B}-\boldsymbol{x}$. Die Constanten zweiter Ordnung sind mit Benutzung der von Reicher angewendeten Formel ${ }^{1}$ ) berechnet worden.

Ein Vergleich mit den Reicher'schen Zahlen ergiebt eine viel stärkere Abweichung der Werthe unter $K_{1}$ und $K_{2}$. Unbestreitbar ist jedenfalls, dass diese Reaction eben kein „ausgezeichneter" Fall einer chemischen Umsetzung ist, die nach einem einfachen Schema verläuft. Dass der Gang der Werthe so viel grösser ist als bei Reicher, ist vermuthlich nicht sowohl auf grössere, durch den raschen Verlauf bei höherer Temperatur bedingte Versuchsfehler zurückzuführen, als vielmehr auf eine mit steigender Temperatur stärker hervortretende Störung des

J) Ostwald, Lehrb. 2, II, 218; Ann. Chem. 228, 257 (1885). 
Bottger n.Kötz: Reaction zw. Chloralhydrat u. Alkali. 485

Vorganges. Ein weiterer Anhaltspunkt dafür wird sich aus dem weiter unten Mitgetheilten über den Einfluss des Verhältnisses von Alkali und Chloralhydrat auf die Temperatur und die Störungen des Vorgangs ergeben.

Um einen Einblick in die störenden Verhältnisse zu bekommen, sind wir in zwei verschiedenen Richtungen an die Prüfung der Frage herangetreten. Wir haben sowohl das Princip, welches der Methode der Isolirung ${ }^{1}$ ) zu Grunde liegt, wie die Bestimmung von $\mathrm{n}$ mit Hülfe der Differential- und der Integralformel (S. 490) auf diese Reaction angewendet. ${ }^{2}$ )

Wir gingen speziell ron der Erwägung aus, dass sich, wenn an der Reaction nur je ein Verbindungsgewicht der beiden fraglichen Stoffe betheiligt ist, der Einfluss der einzelnen Stoffe durch symmetrische Aenderung der Anfangsconcentration des einen, bei Constanz der des anderen, sodass sich bei Ausschluss von Nebenreactionen die gleiche Menge Chloroform gebildet haben würde, am deutlichsten zeigen würde. Setzen sich z. B. die Stoffe $A, B$ nach dem Schema:

um, wobei

$$
A+B=C+D
$$

$$
\frac{d x}{d t}=f\left(C_{A}, C_{B}\right)
$$

ist, so wird man unter Ausschliessung von Nebenreactionen erwarten dürfen, dass die Anfangsgeschwindigkeit bei symmetrischer Veränderung von $C_{A}$ resp。 $C_{B}$ in gleicher Weise verändert wird. Deshalb wird man aus den Abweichungen von dieser Forderung darauf schliessen müssen, dass 1. $A$ oder $B$ sich als positiver oder negativer Katalysator bethätigen kann, oder 2. dass $A$ oder $B$ mit dem Lösungsmittel oder mit den entstehenden Stoffen reagirt oder 3. dass die Anfangsgeschwindigkeit nicht eine Function der analytischen Concentration, sondern eines Veränderungs- (Polymerisations- oder Dissociations-)produktes ist.

Wegen der näheren Verhältnisse, die stattfinden, wenn es sich um Fälle mit Nebenreactionen oder um katalystische Reactionen handelt, verweisen wir auf die Ausführungen von

1) Ostw a ld, Lehrb. 2, II, 238.

2) van't Hoff, Etudea, S. 87. 
486 Bottger u. Kotz: Reaction zw. Chloralhydrat u. Alkali.

Prof. Ostwald. ${ }^{1}$ ) Eine unsymmetrische Beeinflussung der Anfangsgeschwindigkeit wird wegen der angedeuteten Veränderung sehr häufig eintreten. Jedenfalls ist aber zu erwarten, dass trotzdem eine Erhöhung der Concentration eines der Stoffe eine Vergrösserung der Geschwindigkeit bewirken muss. Ist das Gegentheil der Fall, so muss man daraus schliessen, dass eine "Verminderung" der Concentrationen der an der Umsetzung activ betheiligten Ionen oder Stoffe stattgefunden hat. Dass dies wohl möglich ist, ergiebt sich aus den unter diesen Gesichtspunkten angestellten Versuchen, die zunächst mitgetheilt werden sollen.

Ueber die Tabellen ist etwa nur zu erwähnen, dass unter „Titer" die Zahl der zur Neutralisation der unverbrauchten $\mathrm{NaOH}$ nöthigen $\mathrm{cm}^{3}$ Säure angegeben sind. Daraus ergiebt sich mit Berücksichtigung des Neutralisationswerthes des Chloralhydrats der corrigirte Werth $x$, welcher der Umsetzung entspricht, in $\mathrm{Cm} .{ }^{3}$ der benutzten Säure.

\section{Tabelle 2.}

Angewandte Menge Chloralhydrat 0,8920 Grm. in 299,4 Cm. ${ }^{3}$ Angewandte Menge $\mathrm{OH} 0,09379 \mathrm{Grm}$. in $299,4 \mathrm{Cm}^{3}$

$$
\text { Verbältniss in Verbindungsgewichten } \frac{\mathrm{NaOH}}{\mathrm{CCl}_{3} \mathrm{CO}_{2} \mathrm{H}_{3}}=1,083 \text {. }
$$

Berechneter Anfangstiter 10,31, Endtiter 0,79; $a=10,31-0,79=9,52$.

\begin{tabular}{rrrrr} 
Nr. & \multicolumn{1}{c}{$t$} & Titer & $x$ corrig. & $x / a$ \\
1. & 1,1 & 7,77 & 2,47 & 0,26 \\
2. & 2,5 & 5,48 & 5,79 & 0,61 \\
3. & 4,5 & 4,03 & 6,25 & 0,66 \\
4. & 6,0 & 3,16 & 7,13 & 0,75 \\
5. & 7,5 & 2,64 & 7,65 & 0,80 \\
6. & 13,0 & 1,56 & 8,74 & 0,92 \\
7. & 17,7 & 1,15 & 9,16 & 0,96 \\
8. & 23,7 & 0,89 & 9,42 & 0,99 \\
9. & 54 & 0,51 & 9,80 & 1,03 \\
10. & 533 & 0,40 & 9,91 & $1,04$.
\end{tabular}

\section{Tabelle 3a.}

Angewendete Menge Chloralhydrat 1,6840 Grm. in 299,4 Cm. ${ }^{3}$ Angewendete Menge $\mathrm{OH} 0,08932 \mathrm{Grm}$. in $299,4 \mathrm{Cm}^{3}$

1) W. Ostwald, Lehrb. der allg. Chemie 2, II, S. 249-263. 
Böttger u. Kötz: Reaction zw. Chloralhydrat u. Alkali. 487

$\begin{array}{rrrrr}\text { Verhältniss in Verbindungsgewichten: } \frac{\mathrm{NaOH}}{\mathrm{CCl}_{3} \mathrm{CO}_{2} \mathrm{H}_{8}} \\ \text { Berechneter } \\ \text { Anfangstiter } & 8,80 . \quad a=8,80 . \\ \text { Nr. } & t & \text { Titer } & x \text { corrig. } & x / a \\ \text { 1. } & 1,0 & 7,58 & 1,03 & 0,12 \\ \text { 2. } & 20 & 5,07 & 3,57 & 0,41 \\ \text { 3. } & 58 & 2,54 & 6,13 & 0,70 \\ \text { 4. } & 89 & 7,65 & 7,03 & 0,80 \\ \text { 5. } & 1030 & -0,18 & 8,88 & 1,01 .\end{array}$

Tabelle $3 b$.

Parallelversuch.

$\begin{array}{rrrrr}\text { 1. } & 0,8 & 8,20 & 0,41 & 0,05 \\ \text { 2. } & 14,0 & 5,92 & \mathbf{2 , 7 1} & 0,31 \\ \text { 3. } & 29,3 & 4,28 & 4,37 & 0,50 \\ \text { 4. } & 45,0 & 3,09 & 5,57 & 0,63 \\ \text { 5. } & 85 & 1,98 & 6,70 & 0,76 \\ \text { 6. } & 195 & 0,46 & 8,23 & 0,94 \\ 7 . & 335 & 0,10 & 8,60 & 0,98 \\ 8 . & 1338 & -0,12 & 8,82 & 1,00 .\end{array}$

Tabelle 4.

Angewendete Menge Chloralhydrat 3,368 Grm. in 299,4 Cm. Angewendete Menge $\mathrm{OH} 0,08932 \mathrm{Grm}$. in $299,4 \mathrm{Cm}^{3}$

Verhältniss in Verbindungsgewichten: $\frac{\mathrm{NaOH}}{\mathrm{CCl}_{3} \mathrm{CO}_{2} \mathrm{H}_{8}}=\frac{1}{3,88}$.

$$
\text { Anfangstiter }=8,80=a \text {. }
$$

$\begin{array}{rcrcc}\text { Nr. } & t & \text { Titer } & x \text { corrig. } & x / a \\ \text { 1. } & 1 & 8,18 & 0,23 & 0,03 \\ \text { 2. } & 15 & 6,58 & 1,85 & 0,21 \\ \text { 3. } & 34,5 & 4,90 & 3,54 & 0,40 \\ \text { 4. } & 53 & 3,94 & 4,82 & 0,51 \\ \text { 5. } & 100 & \mathbf{2 , 6 2} & 5,78 & 0,66 \\ \text { 6. } & 260 & 0,10 & 8,40 & 0,96 \\ \text { 7. } & 419 & -0,24 & 8,74 & 0,99 \\ \text { 8. } & 1319 & -0,42 & 8,92 & 1,01 .\end{array}$

Tabelle 5a.

Angewendete Menge Chloralhydrat 8,420 Grm. in 299,5 Cm. ${ }^{8}$ Angewendete Menge $\mathrm{OH} 0,08923 \mathrm{Grm}$. in 299,5 $\mathrm{Cm}^{3}$

Verhältniss in Verbindungsgewichten: $\frac{\mathrm{NaOH}}{\mathrm{CCl}_{3} \cdot \mathrm{CO}_{2} \mathrm{H}_{3}}=\frac{1}{9,7}$.

Anfangstiter 8,67 $\mathrm{Cm}^{3}$ 
488 Böttger u.Kotz: Reaction zw. Chloralhydrat u. Alkali.

$\begin{array}{ccccc}\text { Nr. } & t & \text { Titer } & x \text { corrig. } & x / a \\ \text { 1. } & 1,3 & 7,77 & 0,09 & 0,01 \\ \text { 2. } & 15,0 & 6,32 & 1,47 & 0,17 \\ \text { 3. } & 31,5 & 5,45 & 2,35 & 0,27 \\ \text { 4. } & 61,0 & 3,66 & 4,16 & 0,48 \\ \text { 5. } & 170 & 0,91 & 6,94 & 0,80 \\ 6 . & 335 & -0,13 & 8,02 & 0,93 \\ 7 . & 1244 & -0,52 & 8,39 & 0,97 \\ 8 . & 3102 & -0,70 & 8,57 & 0,99 .\end{array}$

Tabelle $5 \mathrm{~b}$.

$\begin{array}{rcrrr}\text { 1. } & 1 & 7,30 & 0,49 & 0,06 \\ \text { 2. } & 5,5 & 6,97 & 0,82 & 0,09 \\ \text { 3. } & 66 & 3,41 & 4,42 & 0,51 \\ \text { 4. } & 133 & 1,90 & 5,95 & 0,69 \\ \text { 5. } & 1093 & -0,90 & 8,77 & 1,01 \\ \text { 6. } & 1528 & -0,89 & 8,76 & 1,01 .\end{array}$

Trägt man diese Werthe in der üblichen Weise in ein Coordinatensystem, wie nachfolgende Figur zeigt, ein und er-

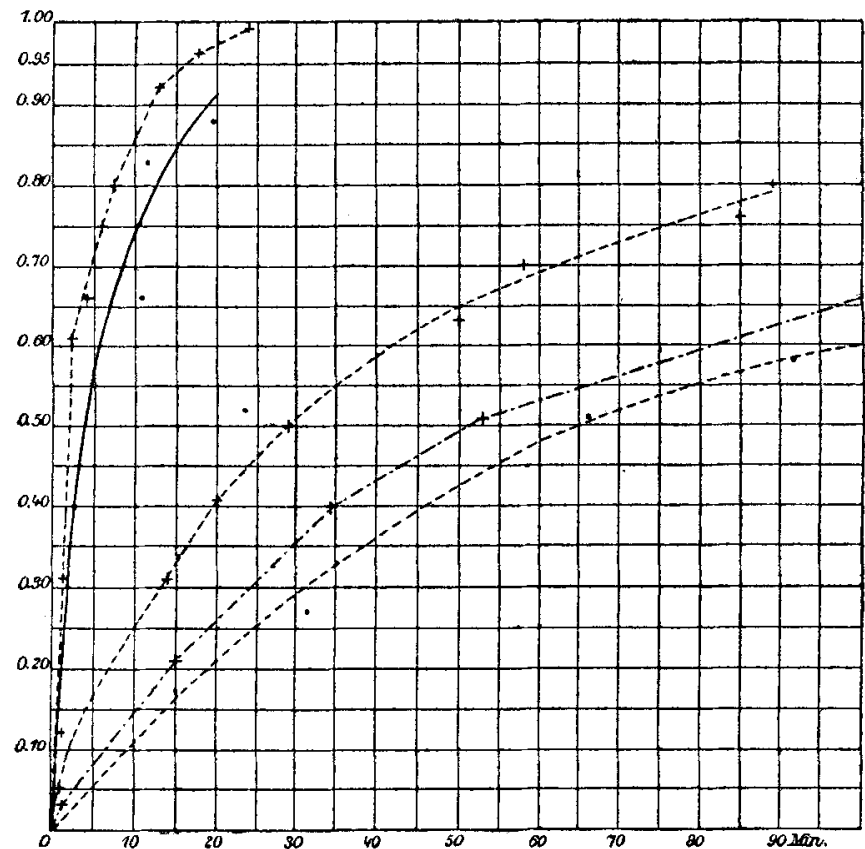

mittelt man die Zeiten, in denen die Hälfte der Umsetzung stattfindet, so ergiebt sich folgende Tabelle: 
Böttger u.Kötz: Reaction zw. Chloralhydrat u. Alkali. 489

Tabelle 6.

\begin{tabular}{|c|c|c|c|c|}
\hline $\begin{array}{l}\text { Normalits } \\
\mathrm{NaOH}\end{array}$ & $\begin{array}{l}\text { am Anfange } \\
\mathrm{CCl}_{3} \mathrm{CO}_{2} \mathrm{H}_{3}\end{array}$ & $\begin{array}{c}\text { Verhältniss } \\
\mathrm{NaOH}: \mathrm{CCl}_{3} \cdot \mathrm{CO}_{2} \mathrm{H}_{3}\end{array}$ & $\begin{array}{l}\text { Zeit für die } \\
1 / 2 \text { Umsetzung }\end{array}$ & $\begin{array}{l}\text { Verb.-Gew. } \\
\mathrm{CCI}_{3} \mathrm{CO}_{2} \mathrm{H}_{3} \\
\text { auf } 1 \mathrm{NaOH}^{-}\end{array}$ \\
\hline 1. 0,0184 & 0,0170 & $1,08: 1$ & $\mathbf{2}^{\prime}$ & 0,926 \\
\hline 2. 0,0175 & 0,0170 & $1,03: 1$ & $4^{\prime}$ & 0,97 \\
\hline 3. 0,0175 & 0,0340 & $1,0: 1,94$ & $29^{\prime}$ & 1,94 \\
\hline 4. 0,0175 & 0,0680 & $1,0: 3,88$ & $5 \mathbf{3}^{\prime}$ & 3,88 \\
\hline 5. 0,0175 & 0,1701 & $1: 9,70$ & $65^{\prime}$ & 9,70 \\
\hline
\end{tabular}

Die Mengen Chloralhydrat in Verbindungsgewichten, die auf $1 \mathrm{NaOH}$ kommen, stehen im Verhältniss:

$$
\text { - } 1: 1,05: 2,10: 4,19: 10,5 \text {, }
$$

Die zur halben Umsetzung nöthigen Zeiten im Verhältniss:

$$
1: 2: 14,5: 26,5: 32,5 \text {. }
$$

Die Discussion dieser Ergebnisse wird nach Mittheilung der anderen Versuche erfolgen. Hier soll nur schon auf den bemerkenswerthen Unterschied hingewiessen werden, den nach Tabelle 6 einerseits die Aenderung der Concentration des Alkalis, andererseits die des Chloralhydrats bedingt.

Diesen Ergebnissen ist noch die Beobachtung anzuschliessen, dass eine sehr concentrirte Lösung von Chloralhydrat, wenn sie nur mit soviel Alkali versetzt ist, dass sie Phenolphtaleïn schwach rosa färbt, nur sehr träge Chloroform bildet. Dagegen tritt eine andere Umsetzung ein, mit der Bildung ron Chlorion verbunden ist, während gleichzeitig die Lösung saure Reaction annimmt. Dieselbe Reaction, auf die noch weiter unten zurückzukommen ist, spielt sich auch in verdünnten Lösungen, wenn Chloralhydrat im Ueberschusse ist, in bemerklichem Betrage ab.

Durch dieselbe erklärt sich ferner die Bedeutung der negativen Werthe in der dritten Columne der Tabelle 3-5. Es soll damit gesagt werden, dass nicht nur kein Alkali mehr vorhanden war, sondern dass sich eine entsprechende Menge Säure gebildet hatte. - Diese Beobachtung deutet zugleich auch auf einen dieGenauigkeit jener Versuche beeinträchtigenden Mangel. Denn die relative Umsetzung wurde unter der Voraussetzung berechnet, dass der Verbrauch von Chloralhydrat 
490 Böttger u.Kötz: Reaction zw. Chloralhydrat u. Alkali.

und Alkali nur nach dem bekannten Schema stattfindet. Da dies nicht der Fall ist, stellen die Versuche richtiger den Verlauf des Gesammt-Alkaliverbrauchs dar. Allerdings ist besonders für den Anfang der Alkaliverbrauch ein Mass der Chloroformbildung, doch ist dieser Umstand bei Ueberschuss an Chloralhydrat im späteren Verlauf bei diesen und ähnlichen Versuchen störend.

\section{Versuche zur Bestimmung von $n$.}

Diese Versuche wurden mit Lösungen ausgeführt, welche äquivalente Mengen von $\mathrm{NaOH}$ und $\mathrm{CCl}_{3} \cdot \mathrm{CO}_{2} \mathrm{H}_{3}$ enthielten. Es wurde bei jedem Versuche die Concentrationsänderung des Alkalis nach zwei Zeiten bestimmt. Die erhaltenen Werthe sind für die 0,01716 n-Lösung:

$$
\begin{gathered}
\text { Tabelle } 7 . \\
-\frac{d r_{1}}{d t}=0,0258 ; \quad-\frac{d c_{2}}{d t}=-0,0170 . \\
t=1 \frac{1}{4}, C \text { (mittl. Conc.) } 8,42 ; \quad t=31 / 2, \quad C=7,58 .
\end{gathered}
$$

Für die 0,00858-n-Ijösung wurde gefunden:

$$
\begin{aligned}
-\frac{d c_{1}{ }^{\prime}}{d t}=0,0087 ; & -\frac{d c_{2}^{\prime}}{d t}=0,0062 . \\
t=11_{4}^{\prime}, C=4,38 ; & t=31_{2}^{\prime}, C=4,04 . \\
n_{1}=1,67 ; & n_{2}=1,60 .
\end{aligned}
$$

Ein Parallelversuch ergab analog:

respective:

$$
\begin{array}{ll}
-\frac{d c_{1}}{d t}=0,0188 ; & -\frac{d c_{2}}{d t}=0,0144 . \\
t=1^{\prime}, C=8,48 ; & t=2^{\prime}, C=7,98,
\end{array}
$$

$$
\begin{array}{rc}
-\frac{d c_{1}{ }^{\prime}}{d t}=0,0064 ; & -\frac{d c_{2}{ }^{\prime}}{d t}=0,0055 . \\
t=1^{\prime}, C=4,39 ; & t=2^{\prime}, C=4,17 . \\
n_{1}=1,70 ; & n=1,41 .
\end{array}
$$

Versuche nach dem Ostwald'schen Princip. $\left.{ }^{1}\right)$

Ferner wurde auch die Beziehung zwischen den Zeiten gleicher relativer Umsetzung und den Geschwindigkeitsfactoren resp. den Concentrationen zur Entscheidung über die Frage

1) Ostwald, Lehrb. 2, II, 235; Z. f. physik. Chem. 2, 134 (1888) und Noyes, Z. f. physik. Ch. 19, 604 (1896). 
Böttger u.Kotz: Reaction zw. Chloralhydrat u. Alkali. 491 herangezogen. Die Ergebnisse sind in der folgenden Tabelle zusammengestellt. Die Bedeutung der einzelnen Columnen ergiebt sich aus den Ueberschriften.

Tabelle 8.

\begin{tabular}{|c|c|c|c|c|c|c|c|}
\hline & \multicolumn{2}{|c|}{ Normalität } & $\begin{array}{c}\text { Verhălniss } \\
\mathrm{NaOH}\end{array}$ & \multirow{2}{*}{$\begin{array}{l}\text { Zeit } \\
\text { in } \\
\text { Sek. }\end{array}$} & \multirow{2}{*}{$\begin{array}{l}\text { Bruch- } \\
\text { theil d. } \\
\text { Um- } \\
\text { setzung }\end{array}$} & \multirow{2}{*}{$\frac{c_{1}}{c_{2}}$} & \multirow{2}{*}{$\frac{t_{2}}{t_{t}}$} \\
\hline & $\mathrm{NaOH}$ & $\mathrm{CCl}_{3} \cdot \mathrm{CO}_{2} \mathrm{H}_{3}$ & $\mathrm{CCl}_{3} \cdot \mathrm{CO}_{2} \mathrm{H}_{3}$ & & & & \\
\hline I. & 1. 0,01716 & 0,01716 & $\frac{1}{1}$ & $107^{\prime \prime}$ & 0,25 & 2 & 1,77 \\
\hline & 2. 0,00858 & 0,00858 & $\frac{1}{1}$ & $189^{\prime \prime *}$ & 0,25 & $\overrightarrow{1}$ & 1 \\
\hline II. & 1. 0,01716 & 0,01716 & $\frac{1}{1}$ & $94^{\prime \prime}$ & 0,25 & 2 & 1,44 \\
\hline & 2. 0,00858 & 0,00858 & $\frac{1}{1}$ & $135^{\prime \prime}$ & 0,25 & $\overline{1}$ & $\overline{1}$ \\
\hline III. & 1. 0,01716 & 0,03432 & $\frac{1}{2}$ & $598^{\prime \prime}$ & 0,20 & 2 & 0,96 \\
\hline & 2. 0,00858 & 0,01716 & $\frac{1}{2}$ & $575^{\prime \prime}$ & 0,20 & $\overline{1}$ & $\overline{1}$ \\
\hline IV. & 1. 0,01802 & 0,01716 & $\frac{1,05}{1}$ & $63^{\prime \prime}$ & 0,25 & 2 & 1,81 \\
\hline & 2. 0,00901 & 0,00858 & $\frac{1,05}{1}$ & $112^{\prime \prime}$ & 0,25 & $\overline{1}$ & $\overline{1}$ \\
\hline
\end{tabular}

Die ziemlich ausgesprochene Abweichung der beiden Parallelversuche I und II dürfte auf eine grössere Unsicherheit des mit einem Stern versehenen Werthes zurückzuführen sein.

\section{Discussion.}

Das Resultat der Beobachtungen ist demnach, dass mit überwiegender Anfangsconcentration des Alkalis die Reaction einen bimolekularen Charakter annimmt. Dagegen ist bei Ueberschuss von Chloralhydrat der monomolekulare Charakter erkennbar. Gleichzeitig treten damit besonders in den späteren Stadien des Verlaufs erhebliche Störungen (Bildung von Chlorion) auf. Bei äquivalenten Mengen ist weder der eine noch der andere Fall rein ausgeprägt, sondern es handelt sich anscheinend um mehrere neben einander verlaufende Vorgänge. Der Uebergang zu einer monomolekularen Reaction bei 
492 Böttger u.Kö tz: Reaction zw. Chloralhydrat u. Alkali.

Erhöhung der Concentration des Chloralhydrats ist jedoch nicht als eine Bestätigung des Erfolges anzusehen, den die Erböhung der Concentration des einen Bestandtheils im allgemeinen hat. ${ }^{1}$ ) Dagegen spricht hier schon die in Tabelle 6 angegebene Verlangsamung. Die unverkennbare Abnahme der Geschwindigkeit des Alkaliverbrauchs mit der Vergrösserung der Anfangsconcentration des Chloralhydrats, die auf eine gegebene Concentration Alkali kommt, deutet darauf hin, dass die Concentration der actuellen Stoffe durch die Vermehrung der potentiellen ${ }^{2}$ ) Concentration der Natronlauge eine Verminderung erfährt. Durch die bereits angeführte Thatsache ${ }^{3}$ ), dass Chloralhydrat sich wie eine sehr schwache Säure verhält und Alkali bis zu gewissem Betrage neutralisirt, wodurch also Verminderung der Concentration des Hydroxylions und Vermehrung der des Chloralhydrations stattfindet, wird die Annahme gestützt, dass das in erster Stufe sich bildende Chloralhydration freiwillig in das stabilere System: Chloroform + Formiation übergeht:

$$
\mathrm{CCl}_{3} \cdot \mathrm{CO}_{2} \mathrm{H}_{2}{ }^{\prime}=\mathrm{CHCl}_{3}+\mathrm{HCO}_{2}{ }^{\prime} \text {. }
$$

Andererseits scheint es nach dem Erfolg der geringen Erhöhung der Concentration des Alkalis, dass das sehr instabile System Chloralhydrat + Alkali auch noch auf andere Weise:

$$
\mathrm{CCl}_{3} \cdot \mathrm{CO}_{2} \mathrm{H}_{3}+\mathrm{OH}^{\prime}=\mathrm{CHCl}_{3}+\mathrm{HCO}_{2}{ }^{\prime}+\mathrm{H}_{2} \mathrm{O}
$$

nach einer bimolekularen Reaction in das stabile System der rechten Seite übergehen kann.

Vermuthlich findet aber die Chloroformbildung auf beiden Wegen statt und nur unter gewissen Grenzbedingungen nach dem einen oder anderen Schema.

Wie verlockend auch eine eingehendere Prüfung der Frage ist, besonders mit Bezug auf die Beantwortung der Frage, ob Hydroxylion katalytisch wirkt, so sind doch die speciellen Verhältnisse so beschaffen, dass die ausgesprochene Deutung der Reaction nicht durch eine exacte Theorie ersetzt werden

1) Ostwald, Lehrb. II, 2. S. $230 . \quad$ 2) Ostwald, s. S. 494.

9) Durch die Beobachtungen M. van Deventer's (Rec. d. trav. chim. des Pays Bas 4, 353 und Maandblad voor Naturwetenschappen 12, 108) wird die Verminderung der activen Menge des Alkalis ebenfalls bestätigt. 
Bottger u.Kotz: Reaction zw. Ohloralhydrat u. Alkali. 493

kann. Die Umstände, die das verhindern, hängen mit der Schwierigkeit, die Hydrolyseconstante des Salzes zu bestimmen, zusammen, ferner mit der Beschränkung auf ein enges Gebiet bei der Untersuchung der Abhängigkeit der Geschwindigkeit der Reaction von der A enderung der Concentration der einzelnen an der Reaction betheiligten Stoffe. Der Steigerung der Concentration des Alkalis ist durch die ausserordentlich starke Vergrösserung der Geschwindigkeit, der Erhöhung der Chloralhydratconcentration durch die erwähnte Nebenreaction eine Grenze gesetzt. Das gebildete Chlorion kann zwar titrirt werden, wenn auch wegen der Reduction des Silbersalzes nur mit ziemlicher Unsicherheit, doch fehlt es zur Berechnung des gebildeten Chloroforms an der Beziehung zwischen Alkaliverbrauch und Chlorionbildung.

Bezüglich der letzteren Umsetzung liegt zunächst die Vermuthung nahe, dass es sich um eine Wechselwirkung zwischen Chloroform und Alkali nach dem bekannten Schema:

$$
\mathrm{CCl}_{3} \mathrm{H}+4 \mathrm{KOH}=3 \mathrm{KCl}+\mathrm{HCO}_{2} \mathrm{~K}+2 \mathrm{H}_{2} \mathrm{O}
$$

handelt. Dieselbe geht jedocb, wie wir uns überzeugt haben, nur bei erheblicher Concentration des Alkalis, da der des Chloroforms durch die geringe Löslichkeit in Wasser eine Grenze gesetzt ist, merklich von statten. Vielmehr fübren andere Beobachtungen, die im nächsten Theil zu erörtern sind, zu der Annahme, dass eine Reaction nach dem Schema:

$$
\mathrm{CCl}_{3} \cdot \mathrm{CO}_{2} \mathrm{H}_{3}+\mathrm{OH}^{\prime}=\mathrm{CCl}_{2} \mathrm{OH} \cdot \mathrm{CO}_{2} \mathrm{H}_{3}+\mathrm{Cl}^{\prime}
$$

vor sich geht.

Ueber den katalytischen Einfluss von Platin auf Reactionen, bei denen Wasser betheiligt ist

$$
\text { (Hydrolyse). }
$$

Bei dem Versuche, die Stärke des Chloralhydrats als Säure durch Leitfähigkeitsmessungen zu bestimmen, stellte sich die schon früher ${ }^{1}$ ) bei anderen Halogenverbindungen be-

1) J. H. van't Hoff und Reicher, Z. f. physik. Chem. 2, 781 (1888); W. Ostwald, das. 3, 176 (1889); M. Wildermann, das. 14, 243 (1894). Seit der Abfassung dieser Abhandlung haben A. Werner und Cl. Herty (Z. f. physik. Chem. 38, 350, 1901) denselben Einfluss von Platinmoor bei der Messung der Leitfähigkeit von Tetrachlorocisdiaminplatin beobachtet. 
obachtete Erscheinung ein, dass die Leitfähigkeit sehr rasch zunimmt, während, wenn die Lösung vorher neutral war, gleichzeitig nach einigem Stehen in Berührung mit Platin die Reactionen auf Wasserstoff- und Chlorion eintreten.

Diese Thatsache hat uns nun veranlasst, diese Erscheiuung nach folgenden Gesichtspunkten zu verfolgen. Es sollte festgestellt werden:

1. In welcher Weise die Geschwindigkeit dieses Vorgangs von der Beschaffenheit des Platins abhängt.

2. In welchem Verhältniss die Menge des gebildeten potentiellen Chlorions ${ }^{1}$ ) und des entstandenen Wasserstoffions stehen.

3. In welcher Weise vorhandenes Chlor- und Wasserstoffion die Reaction beeinflussen.

Nach unseren bisherigen Erfahrungen, die vorwiegend mit Chloralhydrat gemacht wurden, lässt sich die erste Frage kurz dahin beantworten, dass blankes Platinblech eine kaum erkennbare Steigerung der Geschwindigkeit bewirkt. Fein vertheiltes Platin thut dies dagegen in hohem Maasse. Es zeigt sich indessen eine ziemliche Verschiedenheit der Wirkung unter sonst gleichen Umständen verschiedenen Ueberzügen mit Platinschwarz. Ferner hat es den Anschein, dass die Wirksamkeit nach öfterem und längerem Gebrauche abnimmt.

Herr Prof. Bredig war so freundlich, uns von seiner Platintüssigkeit ${ }^{2}$ ) zur Verfügung $\mathbf{z u}$ stellen, um deren Wirkung auf diese Reaction zu ermitteln. Es hat sich dabei herausgestellt, dass mit den gewöhnlichen analytischen Hilfsmitteln die Bildung von Wasserstoff- und Chlorion nach kürzerer Zeit nicht nachgewiesen werden kann. Das Stattfinden einer Umsetzung macht sich jedoch dadurch bemerklich, dass nach einiger Zeit Flockung der Platinflüssigkeit eintritt. Nach längerer Berührung des Platins mit der Flüssigkeit ist die Umsetzung auch durch chemische Mittel nachweisbar. Somit dürften mit Platinschwarz überzogene Bleche die geeignetste Form für die genauere Untersuchung der Erscheinung sein.

Die zweite Frage nach dem Verhältniss des Säure- und

1) W. Ostwald, Grundlinien der anorg. Chemie, S. 252 (1900).

9) Bredig u. Müller von Berneck, Z. f. phys. Chem. 31, 258; Bredig u. Ikeda, 37, 1. 
Böttger u.Kötz: Reaction zw. Chloralhydrat u. Alkali. 495

Chlortiters hängt mit der Frage nach der Natur des Umwandlungsproduktes zusammen. Ist die Annahme richtig, dass der Vorgang in einer Wechselwirkung des Choralhydrats oder anderer Chlorverbindungen mit Wasser, wenigstens in erster Stufe, nach dem Schema:

$$
\mathrm{CCl}_{3} \cdot \mathrm{CO}_{2} \mathrm{H}_{3}+\mathrm{H}_{2} \mathrm{O}=\mathrm{CCl}_{2} \mathrm{OH} \cdot \mathrm{CO}_{2} \mathrm{H}_{8}+\mathrm{HCl} \text {, }
$$

besteht, so darf man vermuthen, dass unter Ausschluss anderer Vorgänge die Mengen des potentiellen Wasserstoff- und Chlorions im Verhältniss 2:1 stehen, wenn die naheliegende Annahme gemacht wird, dass die Gruppe. $\mathrm{CCl}_{2} \mathrm{OH}$ nach Analogie mit . $\mathrm{COOH}$ die Eigenschaften einer Säure hat. ${ }^{1}$ )

Die zur Prüfung dieser Frage angestellten Versuche wurden so ausgeführt, dass ein bekanntes Volum einer $1 / 4$ oder $1 / 16 n$-Lösung von Chloralhydrat mit einem platinirten Bleche in einem verschlossenen Gefässe bestimmte Zeiten hindurch im Thermostat bewegt wurde. Nach entsprechender Dauer wurde die Flüssigkeit abgegossen, das Blech mehrere Male mit Wasser abgespült und die gebildete Säure mit kohlensäurefreier $\mathrm{NaOH}$, das Chlorion mit Silbernitrat titrirt. Die letzteren Titrationen sind wegen der raschen Reduction ziemlich ungenau.

Als Ergebniss einer nicht unbeträchtlichen Reihe vor Versuchen ist zu erwähnen, dass das Verhältniss: Säure zu Chlorion nicht $2: 1$, auch nicht $1: 1$ ist, sondern zwischen diesen Grenzen schwankt, wenn es sich auch in der Mehrzahl der Fälle dem Verbältniss 1:1 nähert. Z. B. ergaben einige Versuche:

Chloralhydrat.

\begin{tabular}{l|c|c|c|c|c|c}
\hline & Dauer & Temp. & $\begin{array}{c}\mathrm{Cm}^{3} \mathrm{NaOH} \\
\frac{1}{26,02} n\end{array}$ & $\begin{array}{c}\mathrm{Cm}^{3} \mathrm{AgNO} \\
\frac{1}{26,02}\end{array}$ & $\begin{array}{c}\text { Quo- } \\
\text { tient }\end{array}$ & $\begin{array}{c}\text { \% des } \\
\text { Gesammt- } \\
\text { chlors }\end{array}$ \\
\hline VI. 20 Cm. ${ }^{3} \frac{1}{4} n$ & $70 \mathrm{St}$. & $25^{0}$ & 31,70 & 24,85 & 1,61 & 6,4 \\
XX. $15 \mathrm{Cm}^{3} \frac{1}{16} n$ & $62 \mathrm{St}$. & $25^{0}$ & 9,05 & 6,11 & $\left.1,48^{2}\right)$ & 8,4 \\
XXI. $15 \mathrm{Cm}^{3} \frac{1}{16} n$ & $62 \mathrm{St}$. & $25^{\circ}$ & 8,73 & 5,86 & $\left.1,49^{2}\right)$ & 8
\end{tabular}

1) Schliesslich wäre auch die in gleichem Sinne sich geltend machende Umlagerung des Reactionsprodukts $\mathrm{zu} \mathrm{CHCl}_{2} \cdot \mathrm{CO}_{2} \mathrm{H}+\mathrm{H}_{2} \mathrm{O}$ in Erwägung zu zieben.

2) Bei einem darauf folgenden Versuch von gleicher Dauer waren die Quotienten 1,31 resp. 1,24 . 
496 Bottger u. Kotz: Reaction zw. Chloralhydrat u. Alkali.

Na-Trichloracetat.

\begin{tabular}{|c|c|c|c|c|c|c|}
\hline$=5+1$ & Dauer & Temp. & $\mid \begin{array}{c}\mathrm{Cm}^{8} \mathrm{NaOH} \\
\frac{1}{26,02} n\end{array}$ & $\begin{array}{c}\mathrm{Cm}^{3} \mathrm{AgNO}_{3} \\
\frac{1}{26,02} n\end{array}$ & $\begin{array}{l}\text { Quo- } \\
\text { tient }\end{array}$ & $\begin{array}{c}\% \text { des } \\
\text { Gesammt } \\
\text { chlors }\end{array}$ \\
\hline II. $15 \mathrm{Cm}^{3} \frac{1}{14,36} n$ & $48 \mathrm{St}$. & $25^{0}$ & 15,40 & 12,68 & 1,21 & 15,50 \\
\hline 1V. $15 \mathrm{Cm} \cdot{ }^{3} \frac{1}{14,36} n$ & 47 St. & $25^{\circ}$ & 7,95 & 6,64 & 1,20 & 8,9 \\
\hline X. $15 \mathrm{Cm}^{3} \frac{1}{46,95} n$ & $48 \mathrm{St}$. & $25^{0}$ & 3,97 & 3,36 & 1,18 & 16,2 \\
\hline
\end{tabular}

Die Erklärung für diesen Befund kann zunächst noch nicht gegeben werden. Möglicherweise ist die Ungenauigkeit der Chlorionbestimmung und wohl auch der verschiedenen Adsorption durch Platin eine Entstellung der wirklichen Verhältnisse zuzuschreiben. Z. B. ergab ein Versuch, bei welchem ein bekanntes Volum mit Salzsäure im Thermostat bewegt wurde, eine Abnahme des Titers.

Die dritte Frage baben wir nur in geringem Umfange in Angriff genommen. Bei zwei Parallelversuchen wurde eine bestimmte Menge $\mathrm{HCl}$ zum Chloralhydrat gegeben und mit zwei verschieden stark wirkenden Blechen bewegt. Dabei wurde in dem einen Falle keine Zunahme, in dem anderen -mit dem wirksameren Bleche - eine deutliche, aber die ohne Zusatz von $\mathrm{HCl}$ beobachtete Reaction nicht überschreitende Zunahme festgestellt. Zu erwarten ist, dass Salzsäure als ein Entstehungsprodukt den Vorgang verlangsamen wird.

Ausser mit Chloralhydrat und trichloressigsaurem Natrium wurde die entsprechende Umsetzung auch mit den Natriumsalzen der Nitro-, m-Cyan-, m-Chlor-, o-Chlor-, Brombenzoë- und Dibrombernsteinsäure qualitativ constatirt. Die Lösung reagirte nach einiger Zeit sauer und zeigte die entsprechenden Reactionen auf $\mathrm{NO}_{2}^{\prime}, \mathrm{CN}^{\prime}, \mathrm{Cl}^{\prime}$ resp. $\mathrm{Br}^{\prime}$. Selbst eine wässrige Lösung von Chloroform, die sonst recht haltbar ist oder richtiger gesagt, die sich ausserordentlich langsam mit Wasser umsetzt, zeigt nach kurzem Stehen mit einem platinirten Platinblech die Kennzeichen jener Umsetzung.

Zwischen dieser Wechselwirkung des Chloralhydrats mit Wasser bei Gegenwart von Platin und der S. 489 erwähnten Nebenwirkung, wenn ein Ueberschuss von Chloralhydrat vor- 
Böttger u.Kötz: Reactionzw. Chloralhydrat u. Alkali. 497

handen ist, besteht nun offenbar ein Zusammenhang. Ohne Platin findet die Reaction bei Zimmertemperatur nur bei einem Ueberschuss von $\mathrm{OH}^{\prime}$ in bemerklichem Betrage statt, bei Anwesenheit von Platin ist dagegen eine so grosse Steigerung der Geschwindigkeit $\mathbf{z u}$ beobachten, dass die Reaction auch trotz der wegen der gebildeten Säure sehr kleinen Concentration des Hydroxylions vor sich geht. Es ist trotz dieses Zusammenhangs indessen ganz wohl möglich, da über die Beständigkeit von $Z$ wischenstufen resp. über die katalytische Wirkung des Platins auf dieselben nichts vorausgesagt werden kann, dass die Produkte der Vorgänge mit und ohne Platin verschieden sind.

Aus äusseren Gründen ist es uns bisher noch nicht möglich gewesen, die Verwirklichung vieler interessanter Vorgänge, wie z. B. die Bildung von Oxalsäure aus Trichloracetat oder auch die des der Dichloressigsäure isomeren Stoffes $\mathrm{CCl}_{2}$. $\mathrm{OH}$.COH zu erreichen. Namentlich der letztere Vorgang oder die Gewinnung der Dichloressigsäure wäre mit Rücksicht auf die Deutung der interessanten Wallach'schen Synthese ${ }^{1}$ ) von Wichtigkeit.

Schliesslich erübrigt es noch, an eine etwaige Betheiligung von Wasserstoff, der vom Platin bei der elektrolytischen Herstellung der Ueberzüge aufgenommen worden sein könnte, zu denken. Es könnte dann nach dem Schema:

$$
\mathrm{CCl}_{3} \mathrm{R}+\mathrm{H}_{2}=\mathrm{CCl}_{2} \mathrm{HR}+\mathrm{HCl}
$$

ebensowohl die Bildung von Wasserstoff und Chlorion verstanden werden. Dies ist zum Beispiel ganz sicher der Fall, wenn in eine mit Wasserstoff in Berührung befindliche Lösung von Trichloressigsäure ein mit Palladium überzogenes Goldblech gebracht wird, wie es der Eine von uns bei dem Versuche, Trichloressigsäure elektrometrisch zu titriren, beobachtet hat. Trotzdem erscheint uns eine Betheiligung des Wasserstofl's aus folgenden Gründen in den oben besprochenen Fällen von geringerem Gewicht zu sein. Wurden die Bleche zur Entfernung von Wasserstoff längere Zeit als Elektroden

1) Wallach, Ber. 9, 1212; 10, 1525 u. 2120. Bis jetzt wird dem Kaliumcyanid, welches mit Chloralhydrat die Ausgangsstoffe bildet, eine katalytische Wirkung zugeschrieben.

Journal $\ell$. prakt. Chemic $[2]$ Bd. 65. 
498 B 8 ttger u.Kotz: Reaction zw. Chloralhydrat u. Alkali.

bei der Elektrolyse verdünnter Schwefelsäure mit Wechselstrom benutzt, so war nach möglichst vollständiger Auswaschung der Bloche kein Unterschied in der Wirksamkeit festzustellen. Ein weiterer, die Annahme der Beschleunigung der „Hydroly se" durch Platin stützender Grund liegt in der Beobachtung, dass auch Platinmohr, der durch Reduction ans Platinchlorid hergestellt wurde und nicht mit $W$ asserstoff in Berührung gekommen war, die Bildung von Chlor- und Wasserstoffion in einer damit in Beruhrung stehenden wässrigen Chloroformlösung bewirkt.

Ferner erfuhren unsere Beobachtungen, die schon vor der Veröffentlichung der Untersuchung von F. Kohlrausch ${ }^{1}$ ) über den Einfluss des Lichts und von Platinmohr auf die Hyilrolyse der Chloride des Goldes, Platins und Zinns vorlagen, durch letztere eine erfreuliche Bestätigung.

Vermuthlich stehen auch die von Raymann und Sulc') beobachteten Wirkungen gewisser Metalle auf die Veränderung der Drehung von Rohrzuckerlösungen mit einer Vergrösserung der Hydrolysegeschwindigkeit in Zusammenhang.

Schliesslich wird die Mittheilung von Interesse sein, dass auch die Verseifung von Aethylacetat bei Gegenwart von Platin rascher erfolgt. Je $15 \mathrm{Ccm}$. einer etwa $1 / 2$ normalen Esterlösung verbrauchten obne resp. mit Platinblech nach 16 Stunden 0,30 resp. $0,94 \mathrm{Ccm}$. ${ }^{p / 28,0}$ : $\mathrm{NaOH}$ zur Neutralisation der gebildeten Säure, also 0,64 Ccm. Natronlauge mehr, wenn die Lösung mit platinirtem Platinblech in Berührung stand.

Auf Grund dieser Versuche und der hier citirten Beobachtungen (für deren Vollständigkeit wir nicht bürgen möchten) halten wir es für eine allgemeine Erscheinung, dass Platinmohr Vorgänge beschleunigt, bei denen Wasser als reagirender Stuff betheiligt ist (Hydrolyse).

Die rorzeitige Veröffentlichung dieser unabgeschlossenen Versuche erfolgt weuiger mit der Absicht, Fachgenossen von der Bearbeitung des Problems fernzuhalten, als aus dem Grunde, weil die Fortsetzung der bisher grösstentheils gemeiusam in Sommer 1900 ausgeführten Versuche durch die räumliche Ent-

i) Kohlrausch, Z. f. physik. Chem. 33, 257 (1900).

2) Reymann u. Sulc, das. 21, 481 (1896). 
Biehringer u. T'opaloff: Ueber Thiopyronin. 499 fernung der Verfasser unerwünschte Verzögerungen erleiden würde.

Herrn Geheimrath Prof. Dr. Ostwald sprechen wir für die Ermöglichung der gemeinsamen Erledigung des hier Mitgetheilten unseren besten Dank aus.

Mit dงr vollständigeren Aufklärung der oben (S. 494) aufgeworfenen Fragen wird sioh der Eine von uns befassen, der Andere (K.) wird die Wirkung von Basen und Säuren auf solche Verbindungen untersuchen, bei denen man an benachbarten Kohlenstoffatomen Sauerstoff oder Halogen gebunden annimmt.

Leipzig und Göttingen, März 1902.

\title{
Ueber Thiopyronin;
}

\author{
von \\ Joachim Biehringer und Wassil Topaloff. ${ }^{1)}$
}

Erster Theil.

Im Jahre 1892 hat die Firma J. R. Geigy \& Co. in Basel ${ }^{2)}$ ein Patent „zur Darstellung blaurother schwefelhaltiger Farbstoffe aus Tetraalkyldiamidodiphenylmethanen" genommen, welche ihrer Bildungsweise und ihrem Verhalten nach als Pyronine aufzufassen sind, die an Stelle des Sauerstoffatoms im Pyronring Schwefel enthalten und demgemäss als "Thiopyronine" bezeichnet werden. In den Handel sind sie unseres Wissens nicht gekommen.

Ueber eine eingehendere Ontersuchung der Farbstoffgruppe, wobei wir uns indessen auf das Tetramethylderivat beschränkt haben, soll im Folgenden berichtet werden.

1) Vgl. J. Biehringer, Ueber Farbstoffe der Pyroningruppe. Dies. Journ. [2] 54, 217 (1896).

2) D.R.P. Nr. 65739 vom 20. Februar 1892 ab. - Friedländer, Fortschritte der Theerfarbenfabrikation, 3, Theil, 1890-1894, S. 97. 